\title{
CONCENTRAÇÃO E ÉPOCA DE APLICAÇÃO DE AMINOETOXIVINILGLICINE (AVG) NA MATURAÇÃO DE MACIEIRAS 'FUJI SUPREMA'’
}

\author{
JOSÉ LUIZ PETRI ${ }^{2}$, FERNANDO JOSÉ HAWERROTH ${ }^{3}$, \\ GABRIEL BERENHAUSER LEITE ${ }^{4}$, MARCELO COUTO ${ }^{4}$
}

RESUMO - O efeito de aminoetoxivinilglicine (AVG), comercialmente disponível com o nome de Retain ${ }^{\circledR}$, foi avaliado em macieiras da cultivar Fuji Suprema durante quatro ciclos agrícolas. Foram estudadas a época de aplicação e a concentração em uma ou em múltiplas aplicações, visando a avaliar o efeito no retardamento da maturação dos frutos, queda pré-colheita dos frutos, produção de frutos, massa média de frutos, resistência da polpa, conteúdo de sólidos solúveis, acidez titulável, índice de iodo-amido, cor da epiderme dos frutos, incidência de pingo-de-mel, incidência de rachadura peduncular e danos de sol. O AVG foi aplicado uma, duas, quatro semanas antes do ponto de colheita e no ponto de colheita, em concentrações variando de 60 a $120 \mathrm{~g} \mathrm{ha}^{-1}$. Todos os tratamentos retardaram a maturação dos frutos, sendo que aplicados duas e uma semana antes do ponto de colheita concentraram a mesma na última data de colheita. $\mathrm{O}$ atraso da maturação foi acompanhado da manutenção da resistência da polpa e degradação do amido. A massa média dos frutos aumentou significativamente em comparação ao tratamento-testemunha. O desenvolvimento da coloração vermelha dos frutos foi retardado proporcionalmente ao atraso da maturação. As aplicações de AVG com diferentes concentrações e épocas de aplicação em uma ou duas vezes não diferiram entre si quanto à queda de frutos em pré-colheita na cultivar Fuji, contudo todos os tratamentos com AVG foram significativamente inferiores ao tratamento-testemunha.

Termos para indexação: Malus domestica, regulador de crescimento, manejo da colheita, abscisão de frutos, qualidade de frutos.

\section{CONCENTRATION AND APPLICATION TIME OF AMINOETHOXYVINYLGLICINE (AVG) ON MATURATION OF 'FUJI SUPREMA' APPLES}

\begin{abstract}
The effect of Aminoethoxyvinylglicine (AVG), available commercially as Retain ${ }^{\circledR}$, was evaluated in Fuji Suprema apples during four growing seasons. The application time and the concentration of AVG in a single or multiple sprays were studied to evaluate the effect of AVG on fruit ripening delay, preharvest drop control, fruit production, mean fruit weight, fruit firmness, soluble solids, total iodine-starch, fruit skin color, 'water core' incidence, fruit crack and sun scald incidence. The AVG was applied one, two, four weeks before the harvest point and at harvest time in concentrations ranging from 60 to $120 \mathrm{~g} \mathrm{ha}^{-1}$. All treatments delayed fruit ripening, and applied twice and one week before the harvest point concentrated on the same harvest date. The maturation delay was accompanied by the maintenance of firmness and starch degradation. The mean fruit weight increased significantly compared to the control treatment. The development of fruit red color was delayed in proportion to the maturation delay. The AVG applications with different concentrations and application times in one or two times did not differ in relation to fruit drop before harvest in 'Fuji' apples, but all treatments with AVG were significantly lower than the control treatment. Index terms: Malus domestica, growth regulator, harvest management, fruit abscission, fruit quality.
\end{abstract}

\footnotetext{
${ }^{1}$ (Trabalho 116-10). Recebido em: 06-05-2010. Aceito para publicação em: 05-11-2010.

${ }^{2}$ Eng. Agr., MSc., Epagri - Estação Experimental de Caçador, C.P. 591, 89500-000, Caçador-SC. E-mail: petri@epagri.sc.gov.br ${ }^{3}$ Eng. Agr., Dr., Embrapa Agroindústria Tropical, 60511-110,Fortaleza-CE. E-mail: fernando@cnpat.embrapa.br

${ }^{4}$ Eng. Agr., Dr., Epagri - Estação Experimental de Caçador, C.P. 591, 89500-000, Caçador-SC. E-mails: gabriel@epagri.sc.gov.br, marcelocouto@epagri.sc.gov.br
} 


\section{INTRODUÇÃO}

$\mathrm{O}$ aminoetoxivinilglicine (AVG), comercializado como Retain ${ }^{\circledR}$, tem sido reportado como inibidor da biossíntese do etileno, apresentando potencial para o controle de maturação e redução da queda de frutos em pré-colheita (GREENE, 2003). O AVG reduz a produção de etileno pelo fruto pelo bloqueio da conversão de S-adenosil-metionina (SAM) para ácido 1-carboxi-1-aminociclopropano (ACC), enzima-chave no caminho da biossíntese do etileno (GREENE, 2003), atuando na diminuição da queda dos frutos pré-colheita e no atraso da maturação em macieira (BYERS et al, 2005; GREENE, 2005).

Comercialmente, o AVG é utilizado para o controle da queda de frutos e retardamento da maturação, sendo aplicado quatro semanas antes do ponto de colheita (YUAN; CARBAUGH, 2007). Contudo, os efeitos de AVG no retardamento da maturação e nos índices de firmeza da polpa, cor da película, degradação de amido e acidez dos frutos são variáveis com a cultivar e a concentração de AVG utilizada (CLAYTON et al. 2000). Greene (2005) relata que o AVG retarda igualmente a queda pré-colheita e a maturação dos frutos quando aplicado de 1 a 4 semanas antes da colheita. Chun et al. (1997) verificaram que a época de aplicação apresentou maior influência do que a concentração de AVG utilizada sobre a queda pré-colheita de maçãs da cv Tsugaro.

A colheita de frutos antes da maturação adequada pode levar a uma conservação deficiente, baixa resistência da polpa e reduzida qualidade (YUAN; CARBAUGH, 2007). Neste sentido, o manejo da maturação dos frutos pode ser de grande importância econômica nas condições brasileiras, onde mais de $90 \%$ da produção está concentrada nas cultivares Gala e Fuji. O uso de aminoetoxivinilglicina $(A V G)$ pode melhorar a qualidade dos frutos na frigoconservação pela colheita no ponto adequado, além de reduzir as perdas em pré-colheita. Além disso, o atraso da maturação, proporcionado pela aplicação de AVG, poderá prolongar o período entre a floração e a maturação dos frutos que poderá resultar em incremento na massa média dos frutos, como abordado por Greene (1996), Amarante et al. (2002) e Petri et al. (2007).

O objetivo deste trabalho foi avaliar o efeito da concentração, época de aplicação e parcelamento das aplicações de AVG no atraso da maturação, na qualidade e na queda pré-colheita dos frutos de macieiras 'Fuji Suprema'.

\section{MATERIAL E MÉTODOS}

Foram realizados quatro experimentos em pomares comerciais, no município de Fraiburgo-SC (Lat. 26 46' S, Long $51^{\circ} \mathrm{W}$ ), nos ciclos 2003/2004, 2004/2005, 2005/2006 e 2006/2007.

\section{Experimento 1}

Foram utilizadas macieiras da cultivar Fuji Suprema com 8 anos de idade, enxertadas sobre o porta-enxerto M-7, durante o ciclo 2003/2004. $\mathrm{O}$ experimento consistiu na aplicação de diferentes combinações de concentrações e de épocas de aplicação de Retain ${ }^{\circledR}$, a seguir: 1) testemunha (sem aplicação); 2) 124,5 $\mathrm{g} \mathrm{ha}^{-1}$ de AVG, 4 semanas antes do ponto de colheita (SAPC); 3) 62,25 $\mathrm{g} \mathrm{ha}^{-1} \mathrm{de}$ AVG 4 SAPC + 62,25 $\mathrm{g} \mathrm{ha}^{-1}$ AVG 2 SAPC; 4) $90 \mathrm{~g} \mathrm{ha}^{-1}$ AVG 2 SAPC; 5) 124,5 $\mathrm{g} \mathrm{ha}^{-1}$ AVG 1 SAPC; e 6) 90 $\mathrm{g} \mathrm{ha}^{-1}$ AVG 1 SAPC. O ponto de colheita (PC) foi considerado em 04-04-04.

\section{Experimento 2}

Foram utilizadas macieiras da cultivar Fuji Suprema com 7 anos de idade, enxertadas sobre o porta-enxerto Marubakaido com interenxerto de M-9, durante o ciclo 2004/2005. A combinação das concentrações e das épocas de aplicação de AVG compôs os seguintes tratamentos: 1) testemunha (sem aplicação); 2) $120 \mathrm{~g} \mathrm{ha}^{-1}$ de AVG 2 SAPC; 3) $60 \mathrm{~g} \mathrm{ha}^{-1}$ AVG 2 SAPC $+60 \mathrm{~g} \mathrm{ha}^{-1}$ AVG 1 SAPC; 4) $120 \mathrm{~g} \mathrm{ha}^{-1}$ AVG 1 SAPC; 5) $60 \mathrm{~g} \mathrm{ha}^{-1}$ AVG 1 SAPC; 6) $60 \mathrm{~g} \mathrm{ha}^{-1} \mathrm{AVG} 1 \mathrm{SAPC}+60 \mathrm{~g} \mathrm{ha}^{-1} \mathrm{AVG}$ no PC; e 7) $60 \mathrm{~g} \mathrm{ha}^{-1}$ no PC. O ponto de colheita (PC) foi considerado em 28-03-05.

\section{Experimento 3}

Foram utilizadas macieiras da cultivar Fuji Suprema com 6 anos de idade, enxertadas sobre o porta-enxerto M-9, durante o ciclo 2005/2006. Os tratamentos testados neste experimento foram os mesmos estudados no experimento 2 . O ponto de colheita (PC) foi considerado em 31-03-06.

\section{Experimento 4:}

Foram utilizadas macieiras da cultivar Fuji Suprema com 9 anos de idade, enxertadas sobre o porta-enxerto Marubakaido com interenxerto de M-9, durante o ciclo 2006/2007, sendo testados os seguintes tratamentos: 1) testemunha (sem aplicação); 2) $120 \mathrm{~g} \mathrm{ha}^{-1}$ AVG 3 SAPC; 3) $60 \mathrm{~g} \mathrm{ha}^{-1}$ AVG 3 SAPC + $60 \mathrm{~g} \mathrm{ha}^{-1}$ AVG 2 SAPC; 4) $60 \mathrm{~g} \mathrm{ha}^{-1}$ AVG 2 SAPC + $60 \mathrm{~g} \mathrm{ha}^{-1} \mathrm{AVG}$ no PC; 5) $300 \mathrm{~g} \mathrm{ha}^{-1}$ AVG após primeira colheita $+300 \mathrm{~g} \mathrm{ha}^{-1} \mathrm{AVG}$ após segunda colheita; 6) $90 \mathrm{~g} \mathrm{ha}^{-1}$ AVG 2 SAPC; e 7) $30 \mathrm{~g} \mathrm{ha}^{-1}$ AVG 1 SAPC 
+ $30 \mathrm{~g} \mathrm{ha}^{-1}$ AVG no PC $+30 \mathrm{~g} \mathrm{ha}^{-1}$ AVG 7 dias após o ponto de colheita (DAPC). Foi considerado como ponto de colheita (PC) adequado a data de 27-03-07.

Em todos os experimentos avaliados, foi adicionado o espalhante adesivo siliconado Silweet ${ }^{\mathbb{R}}$ a $0,05 \%$ nos tratamentos com AVG. Como fonte de AVG, foi utilizado o produto comercial Retain ${ }^{\circledR}$, contendo $15 \%$ do ingrediente ativo. Os tratamentos foram aplicados com pulverizador costal motorizado, com bicos D-S, utilizando-se de um volume de calda de $1.000 \mathrm{~L} \mathrm{ha}^{-1}$. A partir do início da maturação dos frutos nas plantas-controle, iniciou-se a coleta semanal de amostras de 5 frutos por planta de cada repetição, para determinar a resistência da polpa, acidez titulável, o conteúdo de sólidos solúveis, o índice de iodoamido e a porcentagem de cor vermelha da epiderme dos frutos. A firmeza da polpa foi medida em dois lados opostos da superfície de cada fruto, em penetrômetro eletrônico com ponteira de $11 \mathrm{~mm}$. A secção equatorial dos frutos foi exposta à solução de I-KI, e através da análise visual, o índice de degradação do amido foi estimado, utilizando escala de 1 a 9 (BENDER; EBERT, 1985), onde 1 e 9 representam mínima e máxima degradação do amido, respectivamente. $\mathrm{O}$ conteúdo de sólidos solúveis foi determinado com o uso de refratômetro com compensação automática de temperatura, e a acidez titulável foi quantificada titulando-se $10 \mathrm{~mL}$ de suco com $0,1 \mathrm{~N}$ de $\mathrm{NaOH}$ até $\mathrm{pH} \mathrm{8,1} \mathrm{com} \mathrm{titulador} \mathrm{automático.} \mathrm{A} \mathrm{porcentagem} \mathrm{de}$ cor vermelha da epiderme dos frutos foi estimada visualmente, através da relação entre a proporção de cor vermelha da película e a superfície total do fruto.

Em 5 plantas por repetição, foi avaliada a queda de frutos, a produção de frutos por planta $\left(\mathrm{kg} \mathrm{planta}^{-1}\right)$, a massa média dos frutos $\left(\mathrm{g} \mathrm{fruto}^{-1}\right)$, incidência de frutos com pingo de mel, incidência de frutos com rachadura peduncular e incidência de frutos com dano de sol.

$\mathrm{O}$ delineamento experimental utilizado nos três experimentos foi blocos casualizados, com cinco repetições, sendo cada unidade experimental composta de duas plantas. Os resultados obtidos foram submetidos à análise de variância, sendo as variáveis significativas pelo teste $\mathrm{F}(\mathrm{p}<0,05)$, comparadas pelo teste Tukey, a 5\% de probabilidade de erro.

\section{RESULTADOS}

\section{Experimento 1}

A aplicação de AVG em uma ou em duas aplicações, independentemente da época de aplicação e concentração, não apresentou diferenças significativas quanto à resistência de polpa, acidez titulável, massa média de frutos e percentagem de frutos com dano de sol. O conteúdo de sólidos solúveis apresentou diferenças significativas somente em 11-05, sendo o tratamento de $90 \mathrm{~g} \mathrm{ha}^{-1}$ AVG aplicado 2 SAPC inferior à testemunha, sem diferir dos tratamentos 2 e 3 (Tabela 1).

O índice de iodoamido apresentou diferenças significativas somente na amostragem de 12-04, quando todos os tratamentos de AVG foram inferiores ao tratamento-controle, não sendo observadas diferenças significativas entre as épocas de aplicação e a concentração de AVG avaliadas (Tabela 1).

Todos os tratamentos de AVG foram superiores ao tratamento-testemunha, sendo que a maior produção por planta foi observada no tratamento AVG 62,25 $\mathrm{g} \mathrm{ha}^{-1} 4$ SAPC + AVG 62,25 $\mathrm{g} \mathrm{ha}^{-1} 2$ SAPC (Tabela 1). Não foram observadas diferenças significativas entre tratamentos quanto à massa média de frutos, embora os tratamentos de AVG tenham sido numericamente superiores à testemunha, mesmo com maior produção. A incidência de pingo de mel e a incidência de frutos com dano de sol foi reduzida pela aplicação de AVG, porém não houve diferença significativa tanto para época de aplicação como para concentração.

A coloração vermelha da epiderme dos frutos apresentou diferenças significativas somente nas avaliações em 05-04 e 11-04 (Tabela 1). Na primeira avaliação, o tratamento de AVG $90 \mathrm{~g} \mathrm{ha}^{-1} 1 \mathrm{SAPC}$ diferiu significativamente do tratamento-testemunha. $\mathrm{Na}$ última avaliação, não houve diferenças significativa entre a testemunha e os tratamentos de AVG, embora alguns tratamentos de AVG tenham-se diferido entre si, como AVG a 124,5 $\mathrm{g} \mathrm{ha}^{-1} 4$ SAPC e AVG a $90 \mathrm{~g} \mathrm{ha}^{-1} 1$ SAPC.

A produção de frutos por planta foi afetada pela aplicação de Retain ${ }^{\circledR}$, sendo que AVG $62,25 \mathrm{~g} \mathrm{ha}^{-1}$ aplicado 4 e 2 SAPC, AVG $90 \mathrm{~g} \mathrm{ha}^{-1} 2$ SAPC e AVG $124,5 \mathrm{~g} \mathrm{ha}^{-1} 1 \mathrm{SAPC}$ diferiram, significativamente, do tratamento-testemunha, o que pode ser atribuído como hipótese ao aumento da massa média dos frutos (Tabela 2). Em contrapartida, não foram observadas diferenças na massa média de frutos, na incidência de frutos com dano de sol e na incidência de frutos com pingo de mel em resposta à aplicação de Retain ${ }^{\circledR}$, indiferentemente da concentração e época utilizada, embora a massa média dos frutos tenha sido numericamente superior ao tratamento-testemunha em todos os tratamentos de Retain.

\section{Experimento 2}

A resistência de polpa foi superior ao tratamento-testemunha para os tratamentos com a aplicação de AVG a $60 \mathrm{~g} \mathrm{ha}^{-1} 2 \mathrm{SAPC}+60 \mathrm{~g} \mathrm{ha}^{-1}$ 1 SAPC nas avaliações de 16-03, 05-04 e 19-04. 
Entretanto, não se observou diferença significativa para os demais tratamentos, embora as demais avaliações tenham sido numericamente superiores à testemunha (Tabela 3).

Observou-se uma redução no conteúdo de sólidos solúveis com a aplicação de AVG, mantendo-se reduzido até cerca de 30 dias após o ponto de colheita do tratamento-testemunha (Tabela 3). Em 05-04, os tratamentos com aplicação de AVG 2 SAPC apresentaram os menores percentuais de sólidos solúveis. Com o retardamento da colheita, houve um incremento no conteúdo de sólidos solúveis, atingindo conteúdos similares aos da testemunha na avaliação em 26-04.

O índice de iodoamido observado neste experimento confirma os resultados do experimento 1, visto que a aplicação de AVG retardou a degradação do amido. Para todos os tratamentos de AVG, foram observadas diferenças significativas com relação ao tratamento-testemunha aos 47 dias após o ponto de colheita. O tratamento de AVG $120 \mathrm{~g} \mathrm{ha}^{-1}$ 2 SAPC atingiu o índice de iodoamido adequado para armazenagem de frutos por longos períodos, entre 5-04 e 12-04, ou seja, de 20 a 27 dias após o tratamento-testemunha, que apresentou o índice de iodoamido adequado para o ponto de colheita em 16-03 (Tabela 3).

Nas plantas tratadas com AVG, observou-se maior produção por planta, comparadas com as do tratamento-testemunha. Já a massa média dos frutos não apresentou diferenças significativas entre os tratamentos, devendo-se ressaltar que as frutas foram todas colhidas na mesma data (Tabela 4).

A aplicação de AVG reduziu significativamente a percentagem de rachadura peduncular e rachaduras na polpa das frutas em comparação com a testemunha. Entretanto, os tratamentos com AVG não diferiram entre si quanto à rachadura peduncular. Com relação às rachaduras na polpa dos frutos, o tratamento com AVG $120 \mathrm{~g} \mathrm{ha}^{-1} 2$ SAPC diferiu significativamente dos demais tratamentos, à exceção de AVG $60 \mathrm{~g} \mathrm{ha}^{-1} 2 \mathrm{SAPC}+60 \mathrm{~g} \mathrm{ha}^{-1}$ AVG 1 SAPC, reduzindo em $64,6 \%$ as rachaduras em relação ao tratamento-testemunha (Tabela 4).

A aplicação de AVG reduziu significativamente a queda de frutas na pré-colheita. Observou-se o menor percentual de queda de frutas no tratamento de AVG $120 \mathrm{~g} \mathrm{ha}^{-1} 1 \mathrm{SAPC}$ com $1,8 \%$, enquanto na testemunha atingiu $8,9 \%$, com uma diferença de $7,1 \%$ a mais de frutas caídas (Tabela 4).

A ocorrência de escaldadura (dano de sol) apresentou resultados variáveis, embora alguns tratamentos de AVG tenham reduzido a porcentagem de frutas com este dano (Tabela 4).
A incidência de pingo de mel foi reduzida em todos os tratamentos de AVG em comparação com a testemunha, porém não diferiu entre as épocas de aplicação e concentrações, sendo que esta redução pode ser atribuída ao retardamento da maturação das frutas (Tabela 4).

\section{Experimento 3}

A resistência de polpa e o conteúdo de sólidos solúveis não apresentaram diferenças significativas entre tratamentos, sendo que somente o índice de iodoamido foi afetado pela aplicação de AVG (Tabela 5). Na concentração de $60 \mathrm{~g} \mathrm{ha}^{-1} 2 \mathrm{SAPC}+60 \mathrm{~g} \mathrm{ha}^{-1}$ 1 SAPC observou-se o maior efeito na degradação de amido.

A incidência de pingo de mel apresentou resultados variáveis, mas a aplicação de AVG 120 $\mathrm{g} \mathrm{ha}^{-1} 2$ SAPC e 1 SAPC reduziram em $42 \%$ a incidência de pingo de mel nos frutos comparados com a testemunha (Tabela 5).

Embora para a massa média das frutas não tenha sido observada diferença significativa, todos os tratamentos de AVG foram numericamente superiores ao tratamento-testemunha, confirmando os resultados de anos anteriores (Tabela 5).

\section{Experimento 4}

A resistência da polpa e o conteúdo de sólidos solúveis foram pouco influenciados pela aplicação de AVG (Tabela 6), independentemente da época de aplicação e da concentração, corroborando os resultados de anos anteriores.

Em 18-04, 22 dias após o ponto de colheita, os tratamentos de AVG $60 \mathrm{~g} \mathrm{ha}^{-1} 2 \mathrm{SAPC}+60 \mathrm{AVG} g$ ha $^{-1}$ no PC, AVG $30 \mathrm{~g} \mathrm{ha}^{-1}$ após a primeira colheita + $300 \mathrm{~g} \mathrm{ha}^{-1}$ AVG após a segunda colheita e AVG $90 \mathrm{~g}$ $\mathrm{ha}^{-1} 2$ SAPC foram os tratamentos em que os frutos apresentavam a maior resistência da polpa, sendo $5,8 \%$ e $7,1 \%$ superiores ao tratamento-testemunha, respectivamente (Tabela 6). O conteúdo de sólidos solúveis observado foi similar ao de resistência da polpa (Tabela 6).

Foi observado um retardo na degradação do amido pela aplicação de AVG, o que já havia sido observado na colheita em 04-04, mantendo-se até a colheita em 18-04. Entretanto, não se observou diferença significativa entre os tratamentos de Retain $^{\circledR}$, embora no tratamento com AVG $120 \mathrm{~g} \mathrm{ha}^{-1} 3 \mathrm{SAPC}$ tenha sido observada uma tendência de menor degradação do amido (Tabela 6).

Houve uma redução da incidência de pingo de mel, pela aplicação de AVG, embora os níveis tenham sido baixos, com $2 \%$ no tratamento-testemunha e nos tratamentos de AVG que variaram de $0,6 \%$ a $2,6 \%$ 
(Tabela 7).

Observou-se uma tendência de redução de rachadura peduncular com a aplicação de AVG, porém não houve diferença significativa entre época de aplicação e concentração. Também não se observou diferença significativa para o peso médio dos frutos, confirmando resultados de anos anteriores.

\section{DISCUSSÃO}

O efeito das aplicações de AVG sobre a resistência da polpa dos frutos foi reduzido, independentemente da época de aplicação e da concentração, contrariando resultados obtidos por Petri et al. (2007) com a cultivar Gala, visto que, para esta cultivar, os tratamentos com AVG mantiveram a firmeza da polpa. O AVG aplicado 3 a 4 SAPC, mesmo em maiores concentrações, mostrou-se similar às aplicações realizadas próximas ao ponto de colheita.

O conteúdo de sólidos solúveis foi pouco influenciado pelas aplicações de AVG, porém em outras cultivares o AVG reduz o conteúdo de sólidos solúveis (PETRI et al., 2006; PETRI et al., 2007). Isto pode estar relacionado à menor produção de etileno que ocorre em frutos de macieiras 'Fuji' em comparação a frutos da cultivar Gala. A redução do conteúdo de sólidos solúveis variou entre os anos, o que pode estar relacionado a condições ambientais diferenciadas durante o ciclo. Também foi observada tendência de maior redução do conteúdo de sólidos solúveis quando AVG foi aplicado 4 SAPC em maiores concentrações.

A degradação do amido foi retardada com a aplicação de AVG em todos os anos, provavelmente devido a um efeito no atraso da maturação, assim como verificado por Schupp e Greene (2004) e Greene (2005), embora a resistência da polpa não tenha sido influenciada. Aplicações realizadas 2 ou 1 SAPC não mostraram diferenças quanto à degradação de amido, assim como a aplicação realizadas 3 ou 4 SAPC, mesmo em maiores concentrações. O parcelamento em duas aplicações não prolongou o período de diminuição na degradação do amido e, portanto, não foi efetivo.

O desenvolvimento da coloração vermelha da epiderme dos frutos não foi afetado pela aplicação de AVG, diferindo dos resultados obtidos por Amarante et al. (2002), Steffens (2005) e Amarante e Steffens (2009) para a cultivar Gala, na qual observaram a redução na cor vermelha dos frutos pela aplicação de AVG.
A aplicação de AVG na cv. Fuji reduziu substancialmente a ocorrência de pingo de mel e rachadura peduncular. Estas duas variáveis estão relacionadas à colheita dos frutos sob condições inadequadas, ou seja, após o ponto de colheita adequado para frigoconservação. Sendo assim, no presente estudo, houve retardamento na maturação dos frutos na cv. Fuji, o que favorece o manejo da colheita, propiciando a ampliação quantitativa e a qualitativa no período de armazenagem, visto que a menor incidência de pingo de mel reduz a degenerescência da polpa durante a armazenagem dos frutos.

As aplicações de AVG com diferentes concentrações e épocas de aplicação em uma ou duas vezes não diferiram entre si quanto à queda de frutos em pré-colheita na cultivar Fuji, contudo todos os tratamentos com AVG foram significativamente inferiores ao tratamento-testemunha. Embora a cv. Fuji seja menos propensa à queda de frutos na précolheita, devido a sua menor produção de etileno em comparação com a cv. Gala, a aplicação de AVG reduziu a queda de frutos na pré-colheita. Segundo Ward et al. (1999), a queda pré-colheita de maçãs é decorrente da ação do etileno, que aumenta a síntese e a atividade da celulase e poligalacturonase na zona de abscisão dos frutos. De acordo com Yuan e Carbaugh (2007), o fluxo de auxina através da zona de abscisão dos frutos parece determinar a sensibilidade do fruto ao etileno, e a concentração endógena de auxinas deve ser reduzida a certos níveis na zona de abscisão para que a mesma ocorra. Aplicações de AVG de uma a duas SAPC controlam a queda prematura de frutos da cv. Fuji, corroborando os dados obtidos por Greene (2005).

Houve redução na percentagem de frutos com dano de sol pela aplicação de AVG, com tendência a ser mais eficiente quando aplicado 2 semanas antes do ponto de colheita. A produção por planta e a massa média dos frutos apresentaram resultados variáveis, com tendência de aumento do peso médio dos frutos, o que também foi observado na cv. Gala (PETRI et al., 2006). 
TABELA 1 - Sólidos solúveis, índice de iodoamido e percentagem de coloração vermelha nos frutos em macieiras 'Fuji Suprema', em resposta à aplicação de AVG. Fraiburgo-SC, 2004.

\begin{tabular}{|c|c|c|c|c|c|c|}
\hline \multirow{3}{*}{ Tratamentos } & \multicolumn{6}{|c|}{ Data de avaliação } \\
\hline & $05-04-04$ & $12-04-04$ & 19-04-04 & $26-04-04$ & $04-05-04$ & $11-05-04$ \\
\hline & \multicolumn{6}{|c|}{ Sólidos solúveis (\%) } \\
\hline 1. Testemunha & $13,8 \mathrm{~ns}$ & $15,1 \mathrm{~ns}$ & 14,4 ns & $15,2 \mathrm{~ns}$ & $15,0 \mathrm{~ns}$ & $15,7 \mathrm{a}$ \\
\hline 2. AVG $124,5 \mathrm{~g} \mathrm{ha}^{-1} 4 \mathrm{SAPC}^{1}$ & 13,7 & 14,9 & 14,3 & 14,6 & 14,8 & $15,7 \mathrm{a}$ \\
\hline $\begin{array}{l}\text { 3. AVG } 62,25 \mathrm{~g} \mathrm{ha}^{-1} 4 \text { SAPC }+ \\
\text { AVG } 62,25 \mathrm{~g} \mathrm{ha}^{-1} 2 \text { SAPC }\end{array}$ & 13,9 & 14,4 & 14,7 & 15,0 & 15,2 & $15,8 \mathrm{a}$ \\
\hline 4. AVG $90 \mathrm{~g} \mathrm{ha}^{-1} 2 \mathrm{SAPC}$ & 13,6 & 14,5 & 14,6 & 15,0 & 14,8 & $15,0 \mathrm{~b}$ \\
\hline 5. AVG $124,5 \mathrm{~g} \mathrm{ha}^{-1} 1 \mathrm{SAPC}$ & 13,7 & 14,8 & 14,7 & 14,6 & 14,6 & $15,5 \mathrm{ab}$ \\
\hline \multirow[t]{2}{*}{ 6. AVG $90 \mathrm{~g} \mathrm{ha}^{-1} 1 \mathrm{SAPC}$} & 14,0 & 14,9 & 14,5 & 14,9 & 14,6 & $15,4 \mathrm{ab}$ \\
\hline & \multicolumn{6}{|c|}{ Índice de iodoamido (1-9) } \\
\hline 1. Testemunha & $4,6 \mathrm{~ns}$ & $7,0 \mathrm{a}$ & $6,8 \mathrm{~ns}$ & $6,6 \mathrm{~ns}$ & $8,5 \mathrm{~ns}$ & $6,2 \mathrm{~ns}$ \\
\hline 2. AVG $124,5 \mathrm{~g} \mathrm{ha}^{-1} 4 \mathrm{SAPC}$ & 4,2 & $4,6 \mathrm{~b}$ & 5,0 & 5,4 & 8,0 & 6,6 \\
\hline $\begin{array}{l}\text { 3. AVG } 62,25 \mathrm{~g} \mathrm{ha}^{-1} 4 \text { SAPC }+ \\
\text { AVG } 62,25 \mathrm{~g} \mathrm{ha}^{-1} 2 \text { SAPC }\end{array}$ & 4,0 & $4,8 \mathrm{~b}$ & 6,3 & 6,5 & 8,2 & 6,4 \\
\hline 4. AVG $90 \mathrm{~g} \mathrm{ha}^{-1} 2 \mathrm{SAPC}$ & 4,4 & $4,7 \mathrm{~b}$ & 5,1 & 6,6 & 8,2 & 6,4 \\
\hline 5. AVG $124,5 \mathrm{~g} \mathrm{ha}^{-1} 1 \mathrm{SAPC}$ & 3,7 & $4,8 \mathrm{~b}$ & 4,7 & 5,4 & 7,3 & 5,8 \\
\hline \multirow[t]{2}{*}{ 6. AVG $90 \mathrm{~g} \mathrm{ha}^{-1} 1 \mathrm{SAPC}$} & 3,9 & $5,4 \mathrm{~b}$ & 5,4 & 6,6 & 8,0 & 6,3 \\
\hline & \multicolumn{6}{|c|}{ Coloração vermelha da epiderme (\%) } \\
\hline 1. Testemunha & $92,0 \mathrm{a}$ & $85,2 \mathrm{~ns}$ & $89,6 \mathrm{~ns}$ & $94,6 \mathrm{~ns}$ & $89,2 \mathrm{~ns}$ & $86,2 \mathrm{abc}$ \\
\hline 2. AVG $124,5 \mathrm{~g} \mathrm{ha}^{-1} 4 \mathrm{SAPC}$ & 88,8 a & 90,4 & 92,0 & 94,0 & 84,4 & 95,6 a \\
\hline $\begin{array}{l}\text { 3. AVG } 62,25 \mathrm{~g} \mathrm{ha}^{-1} 4 \text { SAPC }+ \\
\text { AVG } 62,25 \mathrm{~g} \mathrm{ha}^{-1} 2 \text { SAPC }\end{array}$ & 88,8 a & 89,2 & 90,4 & 94,4 & 85,6 & $92,0 \mathrm{abc}$ \\
\hline 4. AVG $90 \mathrm{~g} \mathrm{ha}^{-1} 2 \mathrm{SAPC}$ & $88,0 \mathrm{a}$ & 90,0 & 90,4 & 88,0 & 86,8 & 84,8 bc \\
\hline 5. AVG $124,5 \mathrm{~g} \mathrm{ha}^{-1} 1 \mathrm{SAPC}$ & $83,2 \mathrm{ab}$ & 80,4 & 91,6 & 93,2 & 84,0 & $93,6 \mathrm{ab}$ \\
\hline 6. AVG $90 \mathrm{~g} \mathrm{ha}^{-1} 1 \mathrm{SAPC}$ & $76,8 \mathrm{~b}$ & 79,6 & 88,8 & 86,4 & 85,6 & $83,6 \mathrm{c}$ \\
\hline
\end{tabular}

${ }^{1} \mathrm{SAPC}$ - Semanas antes do ponto de colheita. ns - não significativo pelo teste $\mathrm{F}$, a $5 \%$ de probabilidade de erro. Médias seguidas de mesma letra na coluna não diferem entre si, pelo teste Tukey, a $5 \%$ de probabilidade de erro.

TABELA 2 - Produção de frutos por planta, massa média dos frutos, percentagem de frutos com dano de sol e percentagem de frutos com pingo de mel em macieiras 'Fuji Suprema', em resposta à aplicação de AVG. Fraiburgo-SC, 2004.

\begin{tabular}{|c|c|c|c|c|}
\hline Tratamentos & $\begin{array}{c}\text { Produção de } \\
\text { frutos por planta } \\
\text {...kg planta }{ }^{-1} \ldots \\
\end{array}$ & $\begin{array}{c}\text { Massa média } \\
\text { dos frutos } \\
\text {...g fruto } \text { frut. }^{-1}\end{array}$ & 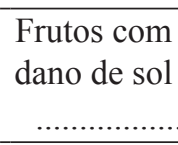 & $\begin{array}{c}\text { Frutos com pingo } \\
\text { de mel } \\
\ldots \%\end{array}$ \\
\hline 1. Testemunha & $12,1 \mathrm{c}$ & $126,5 \mathrm{~ns}$ & $28,2 \mathrm{~ns}$ & $17,0 \mathrm{~ns}$ \\
\hline 2. AVG $124,5 \mathrm{~g} \mathrm{ha}^{-1} 4 \mathrm{SAPC}^{1}$ & $17,5 \mathrm{~b}$ & 135,3 & 28,2 & 7,0 \\
\hline $\begin{array}{l}\text { 3. AVG } 62,25 \mathrm{~g} \mathrm{ha}^{-1} 4 \text { SAPC }+ \\
\text { AVG } 62,25 \mathrm{~g} \mathrm{ha}^{-1} 2 \text { SAPC }\end{array}$ & $32,2 \mathrm{a}$ & 152,2 & 24,8 & 9,0 \\
\hline 4. AVG $90 \mathrm{~g} \mathrm{ha}^{-1} 2 \mathrm{SAPC}$ & $25,5 \mathrm{ab}$ & 153,4 & 26,7 & 10,0 \\
\hline 5. AVG $124,5 \mathrm{~g} \mathrm{ha}^{-1} 1 \mathrm{SAPC}$ & $25,3 \mathrm{ab}$ & 144,6 & 27,0 & 7,0 \\
\hline 6. AVG $90 \mathrm{~g} \mathrm{ha}^{-1} 1 \mathrm{SAPC}$ & $20,7 \mathrm{bc}$ & 142,6 & 28,1 & 6,0 \\
\hline
\end{tabular}

${ }^{1} \mathrm{SAPC}$ - Semanas antes do ponto de colheita. ns - não significativo pelo teste $\mathrm{F}$, a $5 \%$ de probabilidade de erro. Médias seguidas de mesma letra na coluna não diferem entre si, pelo teste Tukey, a 5\% de probabilidade de erro. 
TABELA 3 - Resistência de polpa, sólidos solúveis, índice de iodoamido e percentagem de coloração vermelha nos frutos em macieiras 'Fuji Suprema', em resposta à aplicação de AVG. FraiburgoSC, 2005.

\begin{tabular}{|c|c|c|c|c|c|c|}
\hline \multirow{2}{*}{ Tratamentos } & \multicolumn{6}{|c|}{ Data de avaliação } \\
\hline & 16-03 & $28-03$ & $05-04$ & $12-04$ & 19-04 & $26-04$ \\
\hline & \multicolumn{6}{|c|}{ Resistência de polpa $\left(\mathrm{lb} \mathrm{cm}^{-2}\right)$} \\
\hline 1. Testemunha & $16,8 \mathrm{~b}$ & $15,3 \mathrm{~ns}$ & $15,0 \mathrm{~b}$ & $15,3 \mathrm{~ns}$ & $14,5 \mathrm{~b}$ & $14,4 \mathrm{~ns}$ \\
\hline 2. AVG $120 \mathrm{~g} \mathrm{ha}^{-1} 2 \mathrm{SAPC}^{1}$ & $17,5 \mathrm{ab}$ & 16,6 & $15,4 \mathrm{ab}$ & 15,3 & $14,4 \mathrm{~b}$ & 14,0 \\
\hline 3. AVG $60 \mathrm{~g} \mathrm{ha}^{-1} 2 \mathrm{SAPC}+\mathrm{AVG} 60 \mathrm{~g} \mathrm{ha}^{-1} 1 \mathrm{SAPC}$ & $18,0 \mathrm{a}$ & 16,8 & $16,0 \mathrm{a}$ & 15,7 & $15,2 \mathrm{a}$ & 14,8 \\
\hline 4. AVG $120 \mathrm{~g} \mathrm{ha}^{-1} 1 \mathrm{SAPC}$ & $17,5 \mathrm{ab}$ & 16,6 & $15,2 \mathrm{~b}$ & 15,7 & $14,6 \mathrm{ab}$ & 14,5 \\
\hline 5. AVG $60 \mathrm{~g} \mathrm{ha}^{-1} 1 \mathrm{SAPC}$ & $17,0 \mathrm{ab}$ & 16,7 & $15,4 \mathrm{ab}$ & 15,0 & $14,6 \mathrm{ab}$ & 14,4 \\
\hline 6. AVG $60 \mathrm{~g} \mathrm{ha}^{-1} 1 \mathrm{SAPC}+\mathrm{AVG} 60 \mathrm{~g} \mathrm{ha}^{-1} \mathrm{PC}^{2}$ & $17,3 \mathrm{ab}$ & 16,9 & $15,4 \mathrm{ab}$ & 15,0 & $14,3 \mathrm{~b}$ & 14,1 \\
\hline \multirow[t]{2}{*}{ 7. AVG $60 \mathrm{~g} \mathrm{ha}^{-1} \mathrm{PC}$} & $17,7 \mathrm{ab}$ & 16,7 & $15,5 \mathrm{ab}$ & 15,4 & $14,7 \mathrm{ab}$ & 14,3 \\
\hline & \multicolumn{6}{|c|}{ Sólidos solúveis (\%) } \\
\hline$\overline{1 . \text { Testen }}$ & $14,3 \mathrm{a}$ & $14,6 \mathrm{a}$ & $14,9 \mathrm{a}$ & $15,7 \mathrm{a}$ & $16,8 \mathrm{a}$ & $16,4 \mathrm{ab}$ \\
\hline 2. AVG $120 \mathrm{~g} \mathrm{ha}^{-1} 2 \mathrm{SAPC}$ & $13,3 \mathrm{bc}$ & $12,9 \mathrm{~d}$ & $13,6 \mathrm{~d}$ & $15,3 \mathrm{~d}$ & $14,9 \mathrm{~d}$ & $15,4 \mathrm{~d}$ \\
\hline 3. AVG $60 \mathrm{~g} \mathrm{ha}^{-1} 2 \mathrm{SAPC}+\mathrm{AVG} 60 \mathrm{~g} \mathrm{ha}^{-1} 1 \mathrm{SAPC}$ & $13,4 \mathrm{bc}$ & $14,0 \mathrm{bc}$ & $13,9 \mathrm{~cd}$ & $15,2 \mathrm{bc}$ & $16,0 \mathrm{bc}$ & $15,6 \mathrm{~cd}$ \\
\hline 4. AVG $120 \mathrm{~g} \mathrm{ha}^{-1} 1 \mathrm{SAPC}$ & $13,4 \mathrm{bc}$ & $13,7 \mathrm{c}$ & $14,6 \mathrm{ab}$ & $15,1 \mathrm{bc}$ & $16,0 \mathrm{bc}$ & $16,3 \mathrm{ab}$ \\
\hline 5. AVG $60 \mathrm{~g} \mathrm{ha}^{-1} 1 \mathrm{SAPC}$ & $13,8 \mathrm{abc}$ & $14,3 \mathrm{ab}$ & $14,6 \mathrm{ab}$ & $15,2 \mathrm{ab}$ & $16,3 \mathrm{ab}$ & $16,5 \mathrm{a}$ \\
\hline 6. AVG $60 \mathrm{~g} \mathrm{ha}^{-1} 1 \mathrm{SAPC}+\mathrm{AVG} 60 \mathrm{~g} \mathrm{ha}^{-1} \mathrm{PC}$ & $13,2 \mathrm{c}$ & $13,7 \mathrm{c}$ & $14,1 \mathrm{bcd}$ & $14,9 \mathrm{c}$ & $15,5 \mathrm{c}$ & $15,9 \mathrm{bc}$ \\
\hline \multirow[t]{2}{*}{ 7. AVG $60 \mathrm{~g} \mathrm{ha}^{-1} \mathrm{PC}$} & $13,9 \mathrm{ab}$ & $14,1 \mathrm{abc}$ & $14,3 \mathrm{abc}$ & $15,7 \mathrm{ab}$ & $16,6 \mathrm{a}$ & $16,3 \mathrm{ab}$ \\
\hline & \multicolumn{6}{|c|}{ Índice de iodoamido (1-9) } \\
\hline 1. Testemunha & $4,1 \mathrm{a}$ & $3,4 \mathrm{a}$ & $5,3 \mathrm{a}$ & $6,3 \mathrm{a}$ & $8,8 \mathrm{a}$ & $8,6 \mathrm{a}$ \\
\hline 2. AVG $120 \mathrm{~g} \mathrm{ha}^{-1} 2 \mathrm{SAPC}$ & $3,0 \mathrm{~b}$ & $2,2 \mathrm{~b}$ & $3,8 \mathrm{c}$ & $4,1 \mathrm{c}$ & $6,6 \mathrm{~b}$ & $5,8 \mathrm{bc}$ \\
\hline 3. AVG $60 \mathrm{~g} \mathrm{ha}^{-1} 2 \mathrm{SAPC}+\mathrm{AVG} 60 \mathrm{~g} \mathrm{ha}^{-1} 1 \mathrm{SAPC}$ & $2,2 \mathrm{~b}$ & $2,4 \mathrm{~b}$ & $3,2 \mathrm{c}$ & $4,5 \mathrm{bc}$ & $5,3 \mathrm{c}$ & $4,8 \mathrm{c}$ \\
\hline 4. AVG $120 \mathrm{~g} \mathrm{ha}^{-1} 1 \mathrm{SAPC}$ & $2,2 \mathrm{~b}$ & $2,4 \mathrm{~b}$ & $3,7 \mathrm{bc}$ & $5,0 \mathrm{abc}$ & $6,4 \mathrm{~b}$ & $6,3 \mathrm{~b}$ \\
\hline 5. AVG $60 \mathrm{~g} \mathrm{ha}^{-1} 1 \mathrm{SAPC}$ & $2,9 \mathrm{~b}$ & $3,2 \mathrm{a}$ & $4,8 \mathrm{ab}$ & $6,3 \mathrm{a}$ & $8,4 \mathrm{a}$ & $5,3 \mathrm{bc}$ \\
\hline 6. AVG $60 \mathrm{~g} \mathrm{ha}^{-1} 1 \mathrm{SAPC}+\mathrm{AVG} 60 \mathrm{~g} \mathrm{ha}^{-1} \mathrm{PC}$ & $2,8 \mathrm{~b}$ & $2,4 \mathrm{~b}$ & $3,0 \mathrm{c}$ & $5,1 \mathrm{abc}$ & $8,1 \mathrm{a}$ & $5,8 \mathrm{bc}$ \\
\hline 7. AVG $60 \mathrm{~g} \mathrm{ha}^{-1} \mathrm{PC}$ & $3,1 \mathrm{ab}$ & $2,7 \mathrm{ab}$ & $3,9 \mathrm{bc}$ & $5,9 \mathrm{ab}$ & $6,8 \mathrm{~b}$ & $6,5 \mathrm{~b}$ \\
\hline
\end{tabular}

${ }^{1} \mathrm{SAPC}$ - Semanas antes do ponto de colheita. ${ }^{2} \mathrm{PC}$ - ponto de colheita. ns - não significativo pelo teste $\mathrm{F}$, a $5 \%$ de probabilidade de erro. Médias seguidas de mesma letra na coluna não diferem entre si, pelo teste Tukey, a 5\% de probabilidade de erro.

TABELA 4 - Produção de frutos por planta, massa média dos frutos, percentagem de frutos com rachadura peduncular, percentagem de frutos com rachadura de polpa, percentagem de frutos com rachaduras, percentagem de queda de frutos, percentagem de frutos com dano de sol e percentagem de frutos com pingo de mel em macieiras Fuji Suprema', em resposta à aplicação de AVG. Fraiburgo-SC, 2005.

\begin{tabular}{|c|c|c|c|c|c|c|c|c|}
\hline \multirow[t]{2}{*}{ Tratamentos } & $\begin{array}{l}\text { Produção } \\
\text { de frutos } \\
\text { por planta }\end{array}$ & $\begin{array}{l}\text { Massa } \\
\text { média } \\
\text { dos frutos }\end{array}$ & $\begin{array}{l}\text { Frutos } \\
\text { com } \\
\text { rachadura } \\
\text { peduncular }\end{array}$ & $\begin{array}{c}\text { Frutos } \\
\text { com } \\
\text { rachadura } \\
\text { na polpa }\end{array}$ & $\begin{array}{c}\text { Frutos } \\
\text { com } \\
\text { rachaduras }\end{array}$ & $\begin{array}{l}\text { Queda } \\
\text { de } \\
\text { frutos }\end{array}$ & $\begin{array}{l}\text { Frutos } \\
\text { com dano } \\
\text { de sol }\end{array}$ & $\begin{array}{c}\text { Frutos } \\
\text { com } \\
\text { pingo } \\
\text { de mel }\end{array}$ \\
\hline & ....\%... & ...g fruto $^{-1} .$. & \multicolumn{6}{|c|}{.$\%$} \\
\hline 1. Testemunha & $15,7 \mathrm{c}$ & $151,2 \mathrm{~ns}$ & $8,1 \mathrm{a}$ & $19,5 \mathrm{a}$ & $27,6 \mathrm{~ns}$ & $8,9 \mathrm{a}$ & $25,0 \mathrm{a}$ & $8,3 \mathrm{a}$ \\
\hline 2. AVG $120 \mathrm{~g} \mathrm{ha}^{-1} 2 \mathrm{SAPC}$ & $20,5 \mathrm{abc}$ & 141,8 & $3,2 \mathrm{~b}$ & $6,9 \mathrm{c}$ & 10,1 & $2,3 \mathrm{~b}$ & $18,5 \mathrm{~b}$ & $1,9 \mathrm{~b}$ \\
\hline $\begin{array}{l}\text { 3. AVG } 60 \mathrm{~g} \mathrm{ha}^{-1} 2 \mathrm{SAPC}+ \\
\text { AVG } 60 \mathrm{~g} \mathrm{ha}^{-1} 1 \mathrm{SAPC}\end{array}$ & 2 & 148 & $3,7 \mathrm{~b}$ & 8 & 1 & b & 18 & $1,9 \mathrm{~b}$ \\
\hline 4. AVG $120 \mathrm{~g} \mathrm{ha}^{-1} 1 \mathrm{SAPC}$ & $22,5 \mathrm{abc}$ & 149,6 & $2,3 \mathrm{~b}$ & $13,0 \mathrm{~b}$ & 15,3 & $1,8 \mathrm{~b}$ & 22 & $1,3 \mathrm{~b}$ \\
\hline 5. AVG $60 \mathrm{~g} \mathrm{ha}^{-1} 1 \mathrm{SAPC}$ & $19,0 \mathrm{bc}$ & 146,2 & $2,0 \mathrm{~b}$ & $18,2 \mathrm{a}$ & 20,2 & $4,3 \mathrm{~b}$ & $22,6 \mathrm{ab}$ & $3,1 \mathrm{~b}$ \\
\hline $\begin{array}{l}\text { 6. AVG } 60 \mathrm{~g} \mathrm{ha}^{-1} 1 \mathrm{SAPC}+ \\
\text { AVG } 60 \mathrm{~g} \mathrm{ha}^{-1} \mathrm{PC}^{2}\end{array}$ & $25,2 \mathrm{ab}$ & 147,8 & $2,7 \mathrm{~b}$ & $12,3 \mathrm{~b}$ & 15,0 & $1,8 \mathrm{~b}$ & $17,8 \mathrm{~b}$ & $1,7 \mathrm{~b}$ \\
\hline 7. AVG $60 \mathrm{~g} \mathrm{ha}^{-1} \mathrm{PC}$ & $20,2 \mathrm{abc}$ & 152,0 & $2,9 \mathrm{~b}$ & $12,7 \mathrm{~b}$ & 15,6 & $3,0 \mathrm{~b}$ & $20,8 \mathrm{ab}$ & $2,3 \mathrm{~b}$ \\
\hline
\end{tabular}

SAPC - Semanas antes do ponto de colheita. ${ }^{2} \mathrm{PC}$ - ponto de colheita. ns - não significativo pelo teste $\mathrm{F}$, a $5 \%$ de probabilidade de erro. Médias seguidas de mesma letra na coluna não diferem entre si, pelo teste Tukey, a $5 \%$ de probabilidade de erro. 
TABELA 5 - Resistência de polpa, sólidos solúveis, índice de iodoamido, massa média dos frutos e incidência de frutos com pingo de mel em macieiras 'Fuji Suprema', em resposta à aplicação de AVG. Fraiburgo-SC, 2006.

\begin{tabular}{|c|c|c|c|c|c|}
\hline \multirow{2}{*}{ Tratamentos } & $\begin{array}{l}\text { Resistência de } \\
\text { polpa }\end{array}$ & $\begin{array}{c}\begin{array}{c}\text { Sólidos } \\
\text { solúveis }\end{array} \\
\end{array}$ & $\begin{array}{l}\text { Índice de } \\
\text { iodoamido }\end{array}$ & $\begin{array}{c}\text { Massa média } \\
\text { dos frutos }\end{array}$ & $\begin{array}{l}\text { Frutos com } \\
\text { pingo de mel }\end{array}$ \\
\hline & $\ldots . .1 \mathrm{~b} \mathrm{~cm}{ }^{-2} \ldots$. & $\ldots . . \% \ldots$ & ----1-9---- & $\ldots g$ fruto $^{-1} .$. & $\ldots . . \% \ldots$ \\
\hline 1. Testemunha & $16,1 \mathrm{a}$ & $16,0 \mathrm{a}$ & $5,2 \mathrm{a}$ & $116,7 \mathrm{~ns}$ & $45,0 \mathrm{ab}$ \\
\hline 2. AVG $120 \mathrm{~g} \mathrm{ha}^{-1} 2 \mathrm{SAPC}^{1}$ & $15,3 \mathrm{ab}$ & $14,7 \mathrm{~b}$ & $4,72 \mathrm{ab}$ & 123,3 & $27,0 \mathrm{~b}$ \\
\hline $\begin{array}{l}\text { 3. AVG } 60 \mathrm{~g} \mathrm{ha}^{-1} 2 \mathrm{SAPC}+ \\
\text { AVG } 60 \mathrm{~g} \mathrm{ha}^{-1} 1 \mathrm{SAPC}\end{array}$ & $15,6 \mathrm{ab}$ & $14,9 \mathrm{~b}$ & $2,9 \mathrm{c}$ & 121,0 & $46,7 \mathrm{ab}$ \\
\hline 4. AVG $120 \mathrm{~g} \mathrm{ha}^{-1} 1 \mathrm{SAPC}$ & $14,6 \mathrm{ab}$ & $15,4 \mathrm{ab}$ & $4,2 \mathrm{ab}$ & 120,3 & $27,0 \mathrm{~b}$ \\
\hline 5. AVG $60 \mathrm{~g} \mathrm{ha}^{-1} 1 \mathrm{SAPC}$ & $13,6 \mathrm{~b}$ & $15,3 \mathrm{ab}$ & $4,5 \mathrm{ab}$ & 122,1 & $44,0 \mathrm{ab}$ \\
\hline $\begin{array}{l}\text { 6. AVG } 60 \mathrm{~g} \mathrm{ha}^{-1} 1 \mathrm{SAPC}+ \\
\text { AVG } 60 \mathrm{~g} \mathrm{ha}^{-1} \mathrm{PC}^{2}\end{array}$ & $14,2 \mathrm{ab}$ & $14,6 \mathrm{~b}$ & $4,4 \mathrm{ab}$ & 130,4 & $42,0 \mathrm{ab}$ \\
\hline 7. AVG $60 \mathrm{~g} \mathrm{ha}^{-1} \mathrm{PC}$ & $14,3 \mathrm{ab}$ & $14,9 \mathrm{~b}$ & $3,8 \mathrm{bc}$ & 132,8 & $60,0 \mathrm{a}$ \\
\hline
\end{tabular}

${ }^{1} \mathrm{SAPC}$ - Semanas antes do ponto de colheita. ${ }^{2} \mathrm{PC}$ - ponto de colheita. ns - não significativo pelo teste $\mathrm{F}$, a $5 \%$ de probabilidade de erro. Médias seguidas de mesma letra na coluna não diferem entre si, pelo teste Tukey, a 5\% de probabilidade de erro.

TABELA 6 - Resistência de polpa, sólidos solúveis, índice de iodoamido e percentagem de coloração vermelha nos frutos em macieiras ‘Fuji Suprema’, em resposta à aplicação de AVG. Fraiburgo-SC, 2007.

Tratamentos

Data de avaliação

\begin{tabular}{c}
\hline 4/abr 12/abr 18/abr \\
\hline $\begin{array}{c}\text { Resistência de } \\
\text { polpa }\left(\mathrm{lb} \mathrm{cm}^{-2}\right)\end{array}$
\end{tabular}

\begin{tabular}{|c|c|c|c|}
\hline 1. Testemunha & $16,2 \mathrm{~ns}$ & $15,9 \mathrm{ab}$ & $15,3 \mathrm{~ns}$ \\
\hline 2. AVG $120 \mathrm{~g} \mathrm{ha}^{-1} 3 \mathrm{SAPC}^{1}$ & 16,4 & $16,1 \mathrm{ab}$ & 15,2 \\
\hline 3. AVG $60 \mathrm{~g} \mathrm{ha}^{-1} 3 \mathrm{SAPC}+\mathrm{AVG} 60 \mathrm{~g} \mathrm{ha}^{-1} 2 \mathrm{SAPC}$ & 16,3 & $17,1 \mathrm{a}$ & 15,5 \\
\hline 4. AVG $60 \mathrm{~g} \mathrm{ha}^{-1} 2 \mathrm{SAPC}+\mathrm{AVG} 60 \mathrm{~g} \mathrm{ha}^{-1}$ no $\mathrm{PC}^{2}$ & 16,4 & $16,5 \mathrm{a}$ & 16,2 \\
\hline 5. AVG $30 \mathrm{~g} \mathrm{ha}^{-1}$ após primeira colheita + AVG $30 \mathrm{~g} \mathrm{ha}^{-1}$ após segunda colheita & a 16,2 & $16,3 \mathrm{ab}$ & 16,4 \\
\hline 6. AVG $90 \mathrm{~g} \mathrm{ha}^{-1} 2 \mathrm{SAPC}$ & 16,0 & $16,2 \mathrm{ab}$ & 16,1 \\
\hline 7. $\mathrm{AVG} 30 \mathrm{~g} \mathrm{ha}^{-1} 1 \mathrm{SAPC}+\mathrm{AVG} 30 \mathrm{~g} \mathrm{ha}^{-1} \mathrm{no} \mathrm{PC}+\mathrm{AVG} 30 \mathrm{~g} \mathrm{ha}^{-1} 7 \mathrm{DAPC}^{3}$ & 15,7 & $15,0 \mathrm{~b}$ & 15,1 \\
\hline
\end{tabular}

Sólidos solúveis $(\%)$

1. Testemunha

2. AVG $120 \mathrm{~g} \mathrm{ha}^{-1} 3 \mathrm{SAPC}$

3. AVG $60 \mathrm{~g} \mathrm{ha}^{-1} 3 \mathrm{SAPC}+\mathrm{AVG} 60 \mathrm{~g} \mathrm{ha}^{-1} 2 \mathrm{SAPC}$

$15,7 \mathrm{ab} \quad 16,0 \mathrm{ab} \quad 15,7 \mathrm{abc}$

14,7 c 16,2 ab $14,7 \mathrm{c}$

$15,4 \mathrm{abc} 15,2 \mathrm{~b} \quad 15,3 \mathrm{abc}$

4. AVG $60 \mathrm{~g} \mathrm{ha}^{-1} 2 \mathrm{SAPC}+\mathrm{AVG} 60 \mathrm{~g} \mathrm{ha}^{-1}$ no PC

16,0 a 15,9 ab 15,6 abc

5. AVG $300 \mathrm{~g} \mathrm{ha}^{-1}$ após primeira colheita + AVG $300 \mathrm{~g} \mathrm{ha}^{-1}$ após segunda colheita15,9 ab 15,7 ab $16,5 \mathrm{a}$

6. AVG $90 \mathrm{~g} \mathrm{ha}^{-1} 2$ SAPC 15,4 abc 15,5 ab 16,2 ab

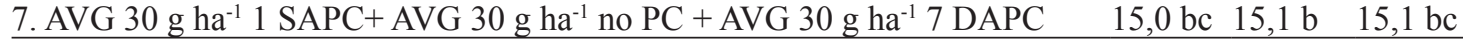

Índice de iodoamido (1-9)

\begin{tabular}{lccc}
\hline 1. Testemunha & $8,6 \mathrm{a}$ & $8,3 \mathrm{a}$ & $8,9 \mathrm{a}$ \\
2. AVG $120 \mathrm{~g} \mathrm{ha}^{-1} 3 \mathrm{SAPC}$ & $5,8 \mathrm{~d}$ & $6,8 \mathrm{c}$ & $7,4 \mathrm{~b}$ \\
3. AVG $60 \mathrm{~g} \mathrm{ha}^{-1}$ 3 SAPC + AVG $60 \mathrm{~g} \mathrm{ha}^{-1}$ 2 SAPC & $6,7 \mathrm{~cd}$ & $7,7 \mathrm{abc}$ & $7,6 \mathrm{~b}$ \\
4. AVG $60 \mathrm{~g} \mathrm{ha}^{-1}$ 2 SAPC + AVG $60 \mathrm{~g} \mathrm{ha}^{-1}$ no PC & $7,9 \mathrm{a}$ & $7,2 \mathrm{bc}$ & $7,6 \mathrm{~b}$ \\
5. AVG $30 \mathrm{~g} \mathrm{ha}^{-1}$ após primeira colheita + AVG $30 \mathrm{~g} \mathrm{ha}^{-1}$ após segunda colheita $7,8 \mathrm{abc}$ & $8,3 \mathrm{a}$ & $8,2 \mathrm{ab}$ \\
6. AVG $90 \mathrm{~g} \mathrm{ha}^{-1}$ 2 SAPC & $7,0 \mathrm{bc}$ & $7,4 \mathrm{abc}$ & $7,5 \mathrm{~b}$ \\
7. AVG $30 \mathrm{~g} \mathrm{ha}^{-1}$ 1 SAPC+ AVG $30 \mathrm{~g} \mathrm{ha}^{-1} \mathrm{no}$ PC + AVG $30 \mathrm{~g} \mathrm{ha}^{-1} 7$ DAPC & $7,2 \mathrm{bc}$ & $8,0 \mathrm{ab}$ & $7,8 \mathrm{ab}$ \\
\hline
\end{tabular}

${ }^{1} \mathrm{SAPC}$ - Semanas antes do ponto de colheita. ${ }^{2} \mathrm{PC}$ - ponto de colheita. ${ }^{3} \mathrm{DAPC}$ - dias após ponto de colheita. ns - não significativo pelo teste $\mathrm{F}$, a $5 \%$ de probabilidade de erro. Médias seguidas de mesma letra na coluna não diferem entre si, pelo teste Tukey, a $5 \%$ de probabilidade de erro. 
TABELA 7 - Massa média dos frutos, percentagem de frutos com rachadura peduncular, percentagem de frutos com pingo de mel em macieiras 'Fuji Suprema', em resposta à aplicação de AVG. Fraiburgo-SC, 2007.

\begin{tabular}{|c|c|c|c|}
\hline \multirow{2}{*}{ Tratamentos } & $\begin{array}{l}\text { Massa média } \\
\text { dos frutos }\end{array}$ & $\begin{array}{l}\text { Frutos com rachadura } \\
\text { peduncular }\end{array}$ & $\begin{array}{c}\text { Frutos com } \\
\text { pingo de mel }\end{array}$ \\
\hline & ---g fruto ${ }^{-1}---$ & \multicolumn{2}{|c|}{-----------\%"---------- } \\
\hline 1. Testemunha & $124,8 \mathrm{~ns}$ & $1,1 \mathrm{~ns}$ & $2,0 \mathrm{~ns}$ \\
\hline 2. AVG $120 \mathrm{~g} \mathrm{ha}^{-1} 3 \mathrm{SAPC}$ & 118,7 & 0,1 & 1,3 \\
\hline 3. AVG $60 \mathrm{~g} \mathrm{ha}^{-1} 3 \mathrm{SAPC}+\mathrm{AVG} 60 \mathrm{~g} \mathrm{ha}^{-1} 2 \mathrm{SAPC}$ & 119,5 & 0,3 & 0,8 \\
\hline 4. AVG $60 \mathrm{~g} \mathrm{ha}^{-1} 2 \mathrm{SAPC}+\mathrm{AVG} 60 \mathrm{~g} \mathrm{ha}^{-1}$ no PC & 119,5 & 0,6 & 2,6 \\
\hline $\begin{array}{l}\text { 5. AVG } 30 \mathrm{~g} \mathrm{ha}^{-1} \text { após primeira colheita }+ \\
\text { AVG } 30 \mathrm{~g} \mathrm{ha}^{-1} \text { após segunda colheita }\end{array}$ & 122,4 & 0,3 & 1,1 \\
\hline 6. AVG $90 \mathrm{~g} \mathrm{ha}^{-1} 2 \mathrm{SAPC}$ & 115,2 & 0,2 & 0,8 \\
\hline $\begin{array}{l}\text { 7. AVG } 30 \mathrm{~g} \mathrm{ha}^{-1} 1 \mathrm{SAPC}+\text { AVG } 30 \mathrm{~g} \mathrm{ha}^{-1} \text { no PC }+ \\
\text { AVG } 30 \mathrm{~g} \mathrm{ha}^{-1} 7 \text { DAPC }\end{array}$ & 118,1 & 0,2 & 0,2 \\
\hline
\end{tabular}

${ }^{1} \mathrm{SAPC}$ - Semanas antes do ponto de colheita. ${ }^{2} \mathrm{PC}$ - ponto de colheita. ${ }^{3}$ DAPC - dias após ponto de colheita. ns - não significativo pelo teste $\mathrm{F}$, a $5 \%$ de probabilidade de erro. Médias seguidas de mesma letra na coluna não diferem entre si, pelo teste Tukey, a $5 \%$ de probabilidade de erro.

\section{CONCLUSÕES}

O uso de AVG na concentração de $60 \mathrm{~g} \mathrm{ha}^{-1}$, aplicado de 2 a 3 SAPC, mostra-se efetivo no manejo da colheita para a cv. Fuji Suprema, permitindo retardar a colheita com manutenção da qualidade dos frutos para armazenagem.

\section{REFERÊNCIAS}

AMARANTE, C.V.T.; SIMIONI, A.; MEGGUER, C.A.; BLUM, L.E.B. Effect of aminoethoxyvinilglicine (AVG) on preharvest fruit drop and maturity of apples. Revista Brasileira Fruticultura, Jaboticabal,, v.24, n.3, p.661-664, 2002.

AMARANTE, C.V.T.; STEFFENS, C.A. O tratamento pré-colheita com $\mathrm{AVG}$, aliado à absorção do etileno durante o armazenamento refrigerado, preserva a qualidade de maçãs 'Gala'. Revista Brasileira de Fruticultura, Jaboticabal, v.31, n.2, p.334-342. 2009.

BENDER, R. J.; EBERT, A. Determinação do ponto de colheita de cultivares de macieira: reste iodoamido. Florianópoli: EMPASC, 1985. 6p.

BYERS, R.E.; CARBAUGH, D.H.; COMBS, L.D. Ethylene inhibitors delay fruit drop, maturity, and increase fruit size of 'Arlet' apples. HortScience, Alexandria, v.40, n.7, p.2061-2065, 2005.
CHUN, J.; PARK, M.; HWANG, Y.; LEE, J. Effect of AVG on preharvest drop and fruit quality in Tsugaru apples. Journal of the Korean Society for Horticultural Science, Suwon, v.38, n.2, p.147-152, 1997.

CLAYTON, M.; BIASI, W.V.; SOUTHWICK, S.M.; MITCHAM, E.J. Retain affects maturity and ripening of 'Bartlett' pear. HortScience, Alexandria, v.35, n.7, p.1294-1299, 2000.

GREENE, D. W. Endogenous hormons and bioregulators use on apple. In: FERRE, D.C.; WARRINGTON, J.J. (Ed.). Apples botany, produtions use. Wallinford: Cabi publishing, 2003. p. 660.

GREENE, D. W. Ethylene based preharvest growth regulators. In: M.A, B.K.M. The fruit physiology growth and development Yakima. Washington: Good Fruit Grower, 1996, p.149-159.

GREENE, D.W. Time of aminoethoxyvinylglycine application influences preharvest drop and fruit quality of 'McIntosh' apples. HortScience, Alexandria, v. 40, n.7, p. 2056-2060, 2005.

PETRI, J.L.; LEITE, G.B.; ARGENTA, L.C. Eficácia do tratamento de AVG no controle da queda e maturação dos frutos de maçã, cultivar Imperial Gala. Revista Brasileira de Fruticultura, Jaboticabal, v.29, p.239-244, 2007. 
PETRI, J. L.; LEITE, G. B.; ARGENTA, L. C.; BASSO, C. Ripening delay and fruit drop control in 'Imperial Gala' and 'Suprema' ('Fuji' Sport) apples by applying AVG (Aminoethoxyvinylglycine). Acta Horticulturae, The Hague, v.727, p.519-525, 2006.

SCHUPP, J.R.; GREENE, D.W. Effect of Aminoethoxyvinylglicine (AVG) on preharvest drop, fruit quality, and maturation of 'McIntosh' apples. I. Concentration and timing of dilute applications of AVG. HortScience, Alexandria, v. 39, p.1030-1035, 2004.

STEFFENS, C.A.; GIEHL, R.F.H.; BRACKMANN, A. Maçã 'Gala' armazenada em atmosfera controlada e tratada com aminoetoxivinilglicina e ethephon. Pesquisa Agropecuária Brasileira, Brasília, v.40, n.9, p.837-843, 2005
WARD, D.L.; BEERS, E.P.; BYERS, R.E.; MARINI, R.P. Cutting apple fruits induces cellulase activity in the abscission zone. HortScience, Alexandria, v.34, n.4, p.601-603, 1999.

YUAN, R.; CARBAUGH, D.H. Effects of ANA, $\mathrm{AVG}$, and 1-MCP on ethylene biosynthesis, preharvest fruit drop, fruit maturity and quality 'Golden Delicious' apples. HortScience, Alexandria, v.42, n.1, p.101-105, 2007. 